out postulating any special solute-solvent interaction.

Department of Chemistry,

NOEL S. BAYLisS

University of Western Australia.

$$
\text { Nov. } 4 .
$$

${ }^{1}$ Kleinberg and Davidson, Chem. Rev., 42, 601 (1948).

${ }^{2}$ Fairbrother, Nature, 160, 87 (1947).

${ }^{3}$ Walker, Trans. Farad. Soc., 31, 1432 (1935).

" Benesi and Hildebrand, J. Amer. Chem. Soc., 70, 2832 (1948).

"Aitkin, Bayliss and Rees, Proc. Roy. Soc., A, 169, 234 (1938).

'Bayliss and Rees, J. Chem. Phys., 8, 377 (1940).

'Rees, J. Chem. Phys., 8, 429 (1940).

${ }^{8}$ Bayliss, Cole and Green; presented to Perth Meeting of Australian and New Zealand Assoc. Adv. Sci., August 1947.

'Green and Rees (unpublished, private communication).

${ }^{10}$ Mulliken and Rieke, Rep. Progr. Physics, 8, 231 (1941).

11 Bayliss ; presented to Perth Meeting of Australian and New Zealand Assoc. Adv. Sci., August 1947, in preparation for publication.

\section{Photoconductivity of Naphthalene and Anthracene}

RECENT studies' of the mobility ('metallic' character) of the unsaturation electrons within molecules containing conjugated double-bond systems suggest that solid conjugated compounds should exhibit the phenomenon of photoconductivity, provided that the unsaturation electrons can acquire enough energy to penetrate the potential barriers between adjoining molecules in the crystal lattice. Photoconductivity has, in fact, been observed in proteins $^{2}$ and in certain dyes ${ }^{3}$. We have found that both naphthalene and anthracene, when melted and allowed to solidify between two platinum electrodes about $0.5 \mathrm{~mm}$. apart, show weak photoconductivity when exposed in vacuo to the unfiltered radiation from a quartz mercury-vapour lamp. With applied voltages between 50 and 360 , the photocurrents are detectable with a good galvanometer. They are of the order of magnitude of $10^{-8} \mathrm{amp}$, and are superimposed on a rather variable dark current. As in the case of dyes ${ }^{8}$, the photocurrents are quenched if air is admitted to the system.

In the case of dyes, the onset and decay of photoconductivity require periods of many minutes ${ }^{3}$, a fact which we confirmed in our preliminary experiments. With naphthalene and anthracene, the onset and decay when the shutter is opened and closed are more rapid than the period $(3 \mathrm{sec}$.) of the galvanometer. By using calibrated wire screens to reduce the incident light intensity, we have found the currents to be directly proportional to the intensity.

The photoconductivity of dyes obeys Ohm's law approximately ${ }^{3}$; but with naphthalene and anthracene the relation between current and voltage is exponential. Within the range of voltage employed, good straight lines are obtained by plotting the logarithm of the current $(i)$ against the voltage $(v)$, suggesting the relation $i=a \exp b v$. This relation cannot hold down to $v=0$, and it suggests comparison with the relation $i=a(\exp b v-1)$ for contacts between metals and semi-conductors. It is not yet possible to say which potential boundary in the system is responsible for the form of the $i-v$ relationship, namely, the boundary between the metal electrode and the organic compound, the inter-crystal boundaries in the molten compound or the inter-molecular boundaries within a single crystal.

The photocurrents are quenched if a fused silica cell containing distilled water is interposed in the path of the light, suggesting that the active radiation is $\lambda .1849$ of the mercury spectrum.

NOEL S. BAYLISS

J. C. RiviERE

Department of Chemistry,

University of Western Australia. Nov. 4.

${ }^{1}$ Bayliss, N. S., J. Chem. Phys., 16, 287 (1948).

2 Szent-Györgyi, A., Nature, 157, 875 (1946).

3 Vartanyan, A. T., J. Phys. Chem. (U.S.S.R.), 20, 1065 (1946).

"Fowler, R. H., "Statistical Mechanics", 433 (Cambridge, 1936).

\section{Effects of Very Fine Solid Particles on Flame Propagation}

METHODS of promoting gaseous combustion reactions are usually of both practical and theoretical significance. Some preliminary observations made by us recently on the apparent influence of very fine solid particles on flame propagation may therefore be of interest.

The particles were introduced into the fuel-air mixture by passing the air (or in some cases its nitrogen constituent), before mixing, through an arc struck between metal electrodes. The mixture was then passed into a long 2-in. diameter vertical glass tube for measurements of limits of inflammability according to the 'flow' method used by Burgoyne and Williams-Leir ${ }^{1}$. The temperature of the gas mixture at the point of application of the igniting source was only a few degrees above atmospheric.

In some cases quite a marked widening of the inflammable range was observed when the arc was in operation. Thus, with iron electrodes and all the air passing through the arc, the lower limit (upward propagation) for hydrogen in air was reduced from $4 \cdot 0$ to $3 \cdot 5$ per cent and that of ethylene from $3 \cdot 4$ to $2 \cdot 7$ per cent. Under the conditions of operation the concentration of iron in the ethylene-air limit mixture was approximately $10^{-2}$ per cent by weight. Electron diffraction patterns indicated that the particles were iron oxide and of the order of $300-$ $500 \mathrm{~A}$. in diameter. Experiments have also been made with methane and with various electrode materials, including carbon. In most, though not in all cases, lower limits are lowered and upper limits raised.

In collaboration with Dr. A. G. Gaydon we have made spectroscopic observations on stationary ethylene-air flames containing particles similarly introduced. The most interesting result is that, with iron electrodes in use, certain Fe lines of high excitation at about $3000 \mathrm{~A}$. occur only in the inner cone of the flame. When copper electrodes are employed, well-marked CN bands are obtained. At present we are studying the chemical effects of the particles on the reactions underlying flame, and consideration is also being given to the physical factors influencing the effects on flame propagation. It would be premature, however, to offer any definite conclusions yet regarding this part of our work.

After we had carried out the preliminary work on limits of inflammability, we realized that some experiments of Lewis and Kreutz ${ }^{2}$ on the subject of 'ionization' are probably relevant to our observations. They found a considerable lowering of the ignition tem. perature of methane-air mixtures the incombustible constituents of which had traversed nickel electrodes across which high-tension sparks were passing. We 